\title{
Avaliação da Tolerabilidade e do Controle de Ciclo de Dois Contraceptivos Orais de Baixa Dose: Estudo Comparativo Aberto.
}

\author{
Assessment of the Tolerability and Cycle Control of Two Low-Dose \\ Oral Contraceptives: An Open-Label Study.
}

\begin{abstract}
Edmund C. Baracat ${ }^{1}$, Ione C. Barbosa ${ }^{2}$, Altamiro A. Campos ${ }^{3}$, Sylvia B. Hyppolito ${ }^{4}$, Nilson R. de $\mathrm{Melo}^{5}$, Rafael Mussielo ${ }^{6}$, Rosival Nassar ${ }^{7}$, João S. Pinho-Neto ${ }^{8}$, Geraldez Tomaz ${ }^{9}$.
\end{abstract}

\section{RESUMO}

Realizou-se um estudo aberto comparativo em nove centros brasileiros para avaliar a tolerabilidade e o controle de ciclo obtido com o uso de dois contraceptivos orais de baixa dose contendo $20 \mathrm{mg}$ etinilestradiol/75 mg gestodeno e $20 \mathrm{mg}$ etinilestradiol/150 mg desogestrel, durante seis ciclos de tratamento. Foram selecionadas 167 mulheres saudáveis com vida sexual ativa (77 no grupo do gestodeno e 90 no grupo do desogestrel), das quais 138 completaram os seis ciclos de tratamento. Em um subgrupo de novas usuárias realizouse também perfil lipídico e hemostático. Foram avaliados 867 ciclos no total. Ocorreu sangramento irregular em 4,6\% dos ciclos com gestodeno e em 8,1\% com desogestrel. A tolerabilidade a ambas preparações foi boa, mas houve significativamente mais náusea no grupo do desogestrel. O controle de ciclo foi bom com os dois contraceptivos, sendo que houve freqüência significativamente menor de sangramento irregular no grupo do gestodeno quando se leva em conta que todos os ciclos foram considerados. Não houve alterações clinicamente significativas no perfil hemostático. O perfil lipídico mostrou tendência a tornarse mais favorável após seis ciclos de tratamento com as duas preparações. Não ocorreu alteração no peso médio das mulheres no grupo do gestodeno; no grupo do desogestrel houve aumento significativo no peso médio de aproximadamente $1 \mathrm{~kg}$ após seis meses de tratamento. A adesão ao tratamento foi boa com as duas preparações. Os resultados deste estudo mostram que preparações contendo baixa dose de gestodeno ou desogestrel associados a $20 \mathrm{mg}$ de etinilestradiol são contraceptivos bem tolerados que permitem bom controle de ciclo, sem efeitos colaterais significantes.

PALAVRAS-CHAVE: Contracepção. Contraceptivo de baixa dose. Gestodeno. Desogestrel. Controle de ciclo. Estrogênio. Progesterona. Coagulação. Perfil lipídico.

\footnotetext{
${ }^{1}$ Escola Paulista de Medicina - Universidade Federal de São Paulo

${ }^{2}$ Faculdade de Medicina da Universidade Federal da Bahia

${ }^{3}$ Hospital Materno-Infantil da Secretaria Estadual de Saúde de Goiás

${ }^{4}$ Faculdade de Medicina da Universidade Federal do Ceará

${ }^{5}$ Faculdade de Medicina da Universidade de São Paulo

${ }^{6}$ Faculdade de Ciências Médicas do Espírito Santo

7 Faculdade de Medicina da Universidade Federal do Pará

8 Faculdade de Medicina da Universidade Federal do

Pernambuco

${ }^{9}$ Faculdade de Medicina da Universidade Federal da Paraíba
}

Trabalho realizado com o apoio dos Laboratórios Wyeth São Paulo.

Correspondência:

Edmund C. Baracat

Rua Botucatu, 572 - cj.102

04023 - 061, São Paulo - SP, Brasil. 


\section{Introdução}

Nas últimas três décadas, o desenvolvimento dos contraceptivos orais evoluiu para diminuição das doses de estrogênio e progestagênio, baseandose no fato de que doses mais baixas estão associadas a menor incidência de alterações metabólicas e de efeitos adversos. Foram empregados novos progestagênios, gestodeno $\mathrm{e}$ desogestrel, pois como agentes com maior seletividade na ação progestagênica podem garantir eficácia contraceptiva e controle de ciclo quando associados a doses mais baixas de etinilestradiol ${ }^{4,6}$.

A dose de gestodeno necessária para inibir a ovulação é menor de que a de outros progestagênios e as preparações contendo gestodeno teriam maior efeito supressor do eixo hipotalâmico-hipofisário ${ }^{6}$.

Com relação ao controle de ciclo e ocorrência de efeitos colaterais, um estudo multicêntrico latino-americano ${ }^{2}$ comparou duas preparações contendo $30 \mathrm{mg}$ de etinilestradiol associados a 75 mg de gestodeno ou a $150 \mathrm{mg}$ de desogestrel. Este estudo mostrou que a associação contendo gestodeno levou a menor incidência de sangramento intermenstrual e de efeitos colaterais indesejáveis. Dados mais recentes ${ }^{1}$ obtidos de estudos comparando preparações contendo $20 \mathrm{mg}$ de etinilestradiol associados a $75 \mathrm{mg}$ de gestodeno ou a $150 \mathrm{mg}$ de desogestrel comprovaram a sua eficácia contraceptiva. No entanto, houve tendência a melhor controle de ciclo e efeito mais favorável sobre a dismenorréia com o udo do gestodeno ${ }^{1}$.

O presente estudo foi realizado para comparar a tolerabilidade e o controle de ciclo de duas preparações contraceptivas orais monofásicas contendo $20 \mathrm{mg}$ de etinilestradiol associados ao gestodeno $75 \mathrm{mg}$ ou desogestrel $150 \mathrm{mg}$ em mulheres brasileiras.

\section{Pacientes e Métodos}

Realizamos um estudo comparativo, aberto, randomizado, multicêntrico, no período de janeiro de 1995 a janeiro de 1998, em nove centros brasileiros. Foram obtidas aprovações dos Comitês de Ética das instituições e o estudo foi conduzido de acordo com a Declaração de Helsinki. Todas assinaram consentimento após informadas sobre o estudo.

Foram elegiveis voluntárias com vida sexual ativa e idades entre 18 e 45 anos (ou 34 para fumantes) que tivessem apresentado ciclos regulares ( 21 a 35 dias) nos três meses anteriores ao início do estudo. Era critério de exclusão a história ou presença de qualquer condição clínica significativa que contra-indicasse o uso de contraceptivos orais ou que pudesse interferir nas avaliações. Além disso, não puderam participar as mulheres que estivessem amamentando ou tivessem utilizado nos últimos seis meses injeções ou implantes de estrogênios, progestagênios ou androgênios.

Cada voluntária foi aleatoriamente selecionada para receber contraceptivos contendo gestodeno $75 \mathrm{mg}$ /etinilestradiol $20 \mathrm{mg}$ (Harmonet* - Wyeth) ou desogestrel $150 \mathrm{mg} /$ etinilestradiol 20 mg (Mercilon* - Organon). Os contraceptivos foram administrados de maneira cíclica (uma drágea diária durante 21 dias consecutivos, seguidos de pausa de 7 dias) por 6 ciclos.

Durante o período de tratamento não era permitido o uso de outros métodos contraceptivos, bem como das seguintes medicações concomitantes: outros hormônios sexuais, anticoagulantes, anticonvulsivantes, isotretinoína, ampicilina, rifampicina, uso crônico de antinflamatórios e sedativos.

\section{Avaliações}

$\mathrm{Na}$ consulta de triagem, foram realizadas as seguintes avaliações: dados demográficos; hábitos (tabagismo); história clínica, ginecológica e obstétrica; exame físico (com medida de peso, altura, pulso e pressão arterial); exame ginecológico; exames hematológico (hemoglobina, hematócrito, contagem de hemácias, leucócitos e plaquetas), bioquímicos (glicemia, uréia, creatinina, cálcio, bilirrubina total, proteína total, albumina, TGO, TGP e fosfatase alcalina), análise de urina e citologia oncótica cervicovaginal.

Um subgrupo das primeiras usuárias foi selecionado aleatoriamente para se efetuar avaliação laboratorial dos sistemas de coagulação e fibrinólise (tempo de protrombina, tempo de tromboplastina parcial ativada, fator VII, fator X, fibrinogênio, antitrombina III, plasminogênio e produtos de degradação da fibrina) e perfil lipídico (colesterol total, HDL, LDL, triglicérides, apolipoproteína A, apolipoproteína B). Estas avaliações foram realizadas no período pré-tratamento e durante a última semana do sexto ciclo.

Os exames físico, ginecológico e laboratoriais foram repetidos na última semana do sexto ciclo de tratamento.

Foram mantidos diários de estudo que eram preenchidos pelas pacientes em todos os ciclos e transcritos pelos investigadores nas respectivas fichas clínicas, nas consultas realizadas no terceiro e no sexto ciclo de tratamento.

Os seguintes sintomas foram acompanhados através de registro nos diários: náusea, cefaléia, enxaqueca, tontura, nervosismo, depressão, fadiga, 
prurido, cloasma, acne, dor abdominal, cãibras, alterações de apetite, alterações de libido, dismenorréia, edema, desconforto mamário, dispareunia, secreção vaginal anormal. A intensidade dos sintomas foi descrita como: 0 - ausente, 1 - leve, 2 - moderada, 3 - intensa.

As pílulas tomadas ou esquecidas foram registradas nos diários durante os seis ciclos. Além disso, foram anotadas no diário a duração e a intensidade do sangramento de privação, duração do período de latência (definido como número de dias entre a última pílula de um ciclo e o início do sangramento), presença de sangramentos de escape (intensidade, duração e período de ocorrência).

\section{Análise Estatística}

A análise estatística foi baseada no conjunto dos dados obtidos de cada centro onde foi realizado o estudo. Realizou-se análise descritiva para todas as variáveis. Os testes estatísticos foram bicaudais e considerou-se como nível de significância $\mathrm{p}<0,05$.

Foram utilizados o teste do $\chi^{2}$ e o teste exato de Fisher para avaliar a equivalência entre os grupos antes do tratamento com relação à distribuição dos atributos nominais. Os atributos com distribuição normal (tais como idade, peso, altura, pulso, pressão arterial e dados laboratoriais) foram comparados pelo teste $\mathrm{t}$ de Student. Os atributos sem distribuição normal, como por exemplo duração e intensidade de fluxo e intensidade dos sintomas foram avaliados pelo teste não-paramétrico de Mann-Whitney. As variações durante o tratamento dos dados laboratoriais foram avaliadas dentro de cada grupo por meio do teste $\mathrm{t}$ de Student para observações pareadas e as comparações entre os grupos foram feitas com o teste t de Student para observações independentes. A evolução dos produtos de degradação da fibrina foi analisada pelo teste de McNemar e os grupos foram comparados pelo teste exato de Fisher. A evolução dos sintomas dentro de cada grupo foi analisada mediante teste sinalizado de Wilcoxon e os grupos foram comparados pelo teste de MannWhitney. Com relação ao número de mulheres apresentando sintomas em cada ciclo e no total de ciclos, a avaliação foi feita usando o teste de $\chi^{2} \mathrm{e}$ teste exato de Fisher. A evolução na duração e intensidade do sangramento de privação dentro de cada grupo foi analisada pelo teste sinalizado de Wilcoxon e a comparação entre os grupos foi feita pelo teste de Mann-Whitney. A comparação entre os grupos com relação à ocorrência de sangramento de escape, bem como com relação à incidência de eventos adversos e descontinuação, foi feita pelo teste do $\chi^{2}$ e teste exato de Fisher.

Foram incluídas no estudo 167 voluntárias, das quais 77 receberam a preparação contendo gestodeno e 90 a com desogestrel. O período de tratamento de seis ciclos foi completado por 138 pacientes, sendo $64(83,1 \%)$ do grupo gestodeno e $74(82,2 \%)$ do grupo desogestrel. Dados de 409 ciclos com gestodeno e 458 ciclos com desogestrel foram analisados. Entre as novas usuárias, um subgrupo de 56 (24 do grupo gestodeno e 32 do grupo desogestrel) foam submetidas a exames de coagulação, fibrinólise e perfil lipídico.

A média de idade foi de 24,3 $\pm 5,2$ anos (média \pm desvio padrão) no grupo gestodeno e 24,6 \pm 5,2 anos no grupo desogestrel. Não houve diferença significante entre os grupos com relação à idade, etnia, tabagismo, peso, dados do exame físico, história ginecológica, uso anterior de contraceptivos orais e características do ciclo menstrual. Também não houve diferença com relação aos exames laboratoriais e aos dados do exame físico entre os grupos antes do início do tratamento.

\section{Resultados}

Com relação aos sintomas pré-mentrais, no grupo gestodeno houve diminuição significativa ( $\mathrm{p}$ $<0,05)$ com relação ao pré-tratamento na intensidade da náusea e da cefaléia (ciclos 4 a 6); nervosismo e desconforto mamário (ciclos 1 a 6); acne (ciclos 2, 3, 5 e 6); dor abdominal (ciclos 1 a 3); dismenorréia (ciclos 3 a 6) e secreção vaginal anormal (ciclos 1 e 2). No grupo desogestrel houve diminuição significativa ( $\mathrm{p}<0,05)$ com relação ao pré-tratamento na intensidade dos seguintes sintomas: nervosismo (ciclo 4); dismenorréia (ciclos 2 a 6); secreção vaginal anormal (ciclos 4 a 6). No grupo desogestrel houve aumento significativo (p $<0,05)$ com relação ao pré-tratamento na intensidade de náusea (ciclos 1 a 3).

Comparativamente entre os grupos, no grupo gestodeno houve diminuição significativa $(p<0,05)$ na incidência de náusea (ciclos 1 a 6), cefaléia (ciclo 6), nervosismo (ciclos 1, 3 e 6), acne (ciclos 2, 3 e 5) e desconforto mamário (ciclos 1, 2 e 3). Considerando-se o total de ciclos, houve incidência significativamente maior de náusea (Figura 1), perda de apetite e cloasma no grupo desogestrel $(p<0,05)$.

\section{Controle de Ciclo}

Em nenhum dos grupos foram observadas alterações clinicamente significativas com relação à duração e intensidade do sangramento de privação. Não houve casos de amenorréia em nenhum dos grupos. O período médio de latência foi de 2,7 \pm 2 dias no grupo gestodeno e de 2,8 \pm 1,9 dias no grupo desogestrel. No grupo gestodeno a incidência de sangramento de escape foi de $9,2 \%$ 
(ciclo 1), 4\% (ciclo 2), 2,7\% (ciclo 3), 6,4\% (ciclo 4), $3,2 \%$ (ciclo 5) e 1,7\% (ciclo 6). Onze mulheres $(14,3 \%)$ relataram sangramento de escape em pelo menos um ciclo. Dos 409 ciclos avaliados, não houve sangramento irregular em 95,4\% dos ciclos e em $4,6 \%$ ocorreu sangramento de escape. A maioria dos episódios de sangramento de escape $(4,4 \%)$ foi do tipo spotting, com duração de 1 a 3 dias $(2,9 \%)$, entre os dias 15 e 21 do ciclo $(2,7 \%)$.

No grupo desogestrel, a incidência de sangramento de escape foi de 11,8\% (ciclo 1), 12\% (ciclo 2), 10,3\% (ciclo 3), 4, 1\% (ciclo 4), 6,9\% (ciclo 5) e $1,5 \%$ (ciclo 6). Dezenove mulheres $(21,1 \%$ ) relataram sangramento de escape em pelo menos um ciclo. Dos 458 ciclos avaliados, não houve sangramento irregular em 91,9\% dos ciclos e em 8,1\% ocorreu sangramento de escape. A maioria dos episódios de sangramento de escape foi do tipo spotting $(7,6 \%)$, com duração de 1 a 3 dias (5,9\%), e período de ocorrência entre os dias 8 e 14 do ciclo $(3,7 \%)$.

A incidência de sangramento de escape foi estatisticamente maior ( $p<0,05)$ no grupo desogestrel, considerando-se o total de ciclos (Figura 2).

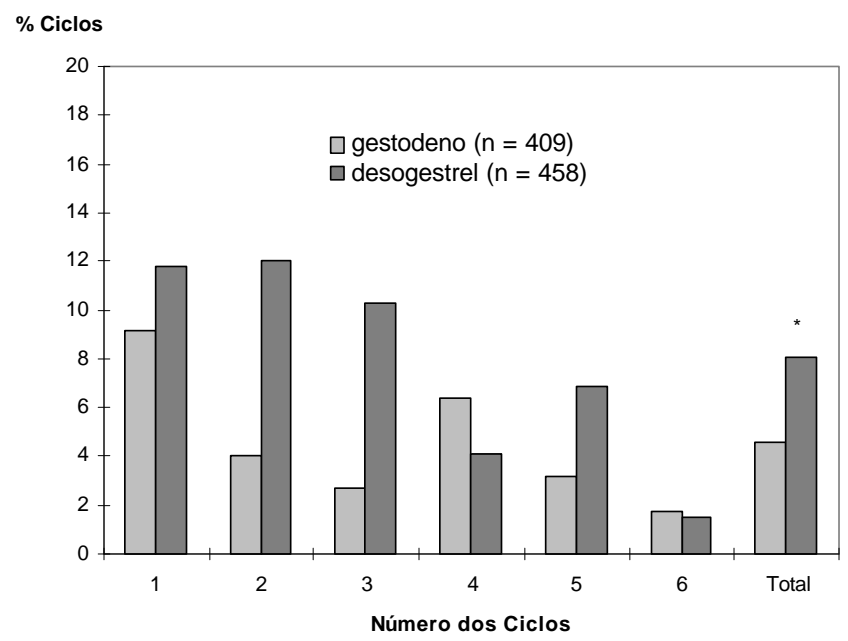

Figura 1 - Porcentagem de ciclos em que foi relatada a ocorrência de náuseas.

* Diferença estatisticamente significante entre os grupos.

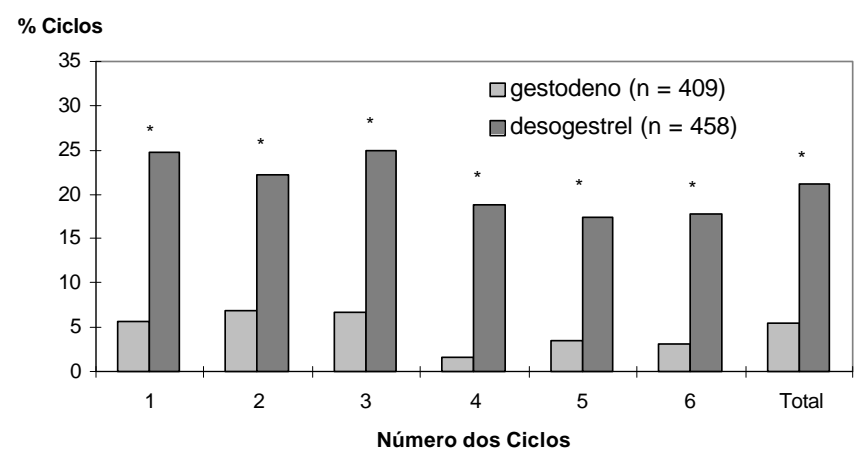

Figura 2 - Porcentagem de ocorrência de sangramento irregular em cada ciclo. * Diferença estatisticamente significante entre os grupos.

\section{Coagulação, Fibrinólise e Perfil Lipídico}

Em nenhum dos grupos houve alteração clinicamente significativa dos fatores de coagulação e fibrinólise avaliados. No grupo gestodeno houve aumento estatisticamente significativo $(\mathrm{p}<0,05)$ no ciclo 6 com relação ao pré-tratamento dos níveis de fator $X$, fibrinogênio, antitrombina III e plasminogênio, que contudo mantiveram-se dentro da faixa de normalidade. No grupo desogestrel houve também aumento estatisticamente significativo $(\mathrm{p}<0,05)$ no ciclo 6 com relação ao pré-tratamento dos níveis de fator X e antitrombina III, que se mantiveram dentro da faixa de normalidade. Considerando-se as variações entre o período pré-tratamento e o ciclo seis, não houve diferença estatisticamente significativa entre os dois grupos (Tabela 1).

Com relação ao perfil lipídico, houve no ciclo 6 com relação ao pré-tratamento aumento no valor médio de HDL no grupo gestodeno de 57 para 62,5 $\mathrm{mg} / \mathrm{dl}(\mathrm{p}=0,069)$ e no grupo desogestrel de 55,6 para 58,9 mg/dl $(\mathrm{p}=0,036)$. No grupo desogestrel houve aumento no valor médio da apolipoproteína $\mathrm{B}$ de 99,5 para $106,1 \mathrm{mg} / \mathrm{dl}(\mathrm{p}=0,046)$. Houve diminuição nos triglicérides no grupo gestodeno e aumento no grupo desogestrel. Embora dentro de cada grupo essa variação não tenha atingido significância estatística, houve diferença significativa $(p=0,011)$ na variação comparandose os dois grupos entre si (Tabela 2).

\section{Peso}

$\mathrm{Na}$ avaliação antes do início do tratamento o peso médio no grupo gestodeno foi de 56,3 \pm 9,0 $\mathrm{kg}$ e, no grupo desogestrel, de 55,6 \pm 9,4 kg.

No grupo gestodeno não houve aumento significativo de peso $(p=0,679)$ com relação ao pré-tratamento em nenhum dos ciclos (peso médio no ciclo 6: 56,2 $\pm 9,1 \mathrm{~kg}$ ). No ciclo 6 , com relação ao pré-tratamento, $26,6 \%$ das mulheres apresentaram ganho de peso inferior a $2 \mathrm{~kg}$ e 10,9\% ganho superior a $2 \mathrm{~kg}$; 32,8\% não apresentaram variação de peso.

Já no grupo desogestrel, houve aumento médio significativo de $1 \mathrm{~kg}$ no ciclo 6 com relação ao valor pré-tratamento $(\mathrm{p}=0,002)$. No ciclo 6 , com relação ao pré-tratamento, $51,4 \%$ das mulheres apresentaram ganho de peso inferior a 2 $\mathrm{kg}$ e $10,8 \%$ ganho superior a $2 \mathrm{~kg} ; 21,6 \%$ não apresentaram variação de peso.

\section{Eventos Adversos e Abandono do Estudo}

No grupo gestodeno, 19,5\% das mulheres relataram um ou mais eventos adversos. Os mais freqüentes foram cefaléia, fadiga e náusea. No grupo desogestrel, 20\% das mulheres relataram um ou mais eventos adversos, dos quais os mais 
freqüentes foram náusea, cefaléia, desconforto mamário e sangramento vaginal.

No grupo gestodeno, 13 mulheres $(16,9 \%)$ abandonaram o estudo, sendo que em 6 a razão para o abandono foi a ocorrência de algum evento adverso. No grupo do desogestrel, 16 mulheres $(17,8 \%)$ abandonaram o estudo, das quais em 8 a razão para o abandono foi a ocorrência de algum evento adverso.

Não houve diferença significativa entre os grupos com relação à incidência de eventos adversos e descontinuação do estudo.

Houve uma gravidez no grupo do desogestrel devido à falha de tomada no segundo ciclo de tratamento.

Não houve alterações clínicas no pulso, pressão arterial, exames físico e laboratoriais (hematológico, bioquímico, urina e citologia oncológica cervicovaginal) nos dois grupos estudados.

$\mathrm{Na}$ avaliação ginecológica ao final do tratamento, o achado mais freqüente nos dois grupos, observado em menos de $2 \%$ das mulheres, foi hiperemia nos genitais externos e ectopia cervical.

A porcentagem de esquecimento de tomada de pílulas foi de $12 \%$ no grupo gestodeno e $10,5 \%$ no grupo desogestrel.

Tabela 1 - Fatores de coagulação e fibrinólise avaliados no pré-tratamento e ao final do sexto ciclo.

\begin{tabular}{|c|c|c|c|c|c|c|c|}
\hline $\begin{array}{l}\text { Fatores de } \\
\text { coagulação e } \\
\text { fibrinólise }\end{array}$ & gestodeno (pré) & $\begin{array}{l}\text { gestodeno } \\
\text { (ciclo 6) }\end{array}$ & & desogestrel (pré) & $\begin{array}{l}\text { desogestrel } \\
\text { (ciclo 6) }\end{array}$ & & $\begin{array}{c}\text { significância } \\
\text { (variação entre } \\
\text { grupos) }\end{array}$ \\
\hline $\begin{array}{l}\text { Tempo de } \\
\text { Protrombina (s) }\end{array}$ & $12,8 \pm 1,4$ & $13,0 \pm 1,4$ & $\mathrm{p}=0,878$ & $13,0 \pm 1,9$ & $12,6 \pm 1,6$ & $\mathrm{p}=0,148$ & $p=0,262$ \\
\hline Fator VII (\%) & $99,6 \pm 11,3$ & $97,5 \pm 12,2$ & $\mathrm{p}=0,286$ & $99,3 \pm 8,3$ & $98,8 \pm 7,6$ & $\mathrm{p}=0,446$ & $\mathrm{p}=0,441$ \\
\hline Fator X (\%) & $95,2 \pm 16,7$ & $97,8 \pm 15,5$ & $* p=0,001$ & $101,2 \pm 17,7$ & $107,0 \pm 17,5$ & $* \mathrm{p}=0,001$ & $\mathrm{p}=0,594$ \\
\hline Plasminogênio (\%) & $101,1 \pm 9,8$ & $104,2 \pm 9,7$ & $* p=0,018$ & $98,7 \pm 12,7$ & $98,6 \pm 10,8$ & $\mathrm{p}=0,924$ & $\mathrm{p}=0,051$ \\
\hline $\begin{array}{l}\text { Produtos de } \\
\text { Degradação da } \\
\text { Fibrina } \\
(\%<2,5 \mu \mathrm{g} / \mathrm{ml})\end{array}$ & 100 & 100 & - & 96,7 & 100 & - & - \\
\hline
\end{tabular}

* Diferenças estatisticamente significativas

APTT: tempo de tromboplastina parcial ativada 
Tabela 2 - Perfil lipídico avaliado no pré-tratamento e ao final do sexto ciclo.

\begin{tabular}{|c|c|c|c|c|c|c|c|}
\hline Lípides & gestodeno (pré) & $\begin{array}{l}\text { gestodeno } \\
\text { (ciclo 6) }\end{array}$ & & desogestrel (pré) & $\begin{array}{l}\text { desogestrel } \\
\text { (ciclo 6) }\end{array}$ & & $\begin{array}{c}\text { Significância } \\
\text { (variação entre grupos) }\end{array}$ \\
\hline $\begin{array}{l}\text { Colesterol Total } \\
(\mathrm{mg} / \mathrm{dl})\end{array}$ & $148,6 \pm 39,7$ & $141,5 \pm 41,62$ & $\mathrm{p}=0,106$ & $155,8 \pm 39,5$ & $157,6 \pm 40,7$ & $\mathrm{p}=0,981$ & $\mathrm{p}=0,136$ \\
\hline HDL (mg/dl) & $57,0 \pm 15,3$ & $62,5 \pm 15,5$ & $\mathrm{p}=0,069$ & $55,6 \pm 12,0$ & $58,9 \pm 11,7$ & $* p=0,036$ & $\mathrm{p}=0,516$ \\
\hline $\mathrm{LDL}(\mathrm{mg} / \mathrm{dl})$ & $78,6 \pm 30,3$ & $81,1 \pm 29,6$ & $\mathrm{p}=0,710$ & $80,4 \pm 33,2$ & $76,9 \pm 32,3$ & $\mathrm{p}=0,431$ & $\mathrm{p}=0,433$ \\
\hline $\begin{array}{l}\text { Triglicérides } \\
(\mathrm{mg} / \mathrm{dl})\end{array}$ & $115,1 \pm 22,7$ & $106,8 \pm 21,8$ & $\mathrm{p}=0,071$ & $111,7 \pm 29,6$ & $115,3 \pm 31,1$ & $\mathrm{p}=0,075$ & $* \mathrm{p}=0,011$ \\
\hline $\begin{array}{l}\text { Apolipoproteína A } \\
(\mathrm{mg} / \mathrm{dl})\end{array}$ & $136,0 \pm 32,6$ & $132,7 \pm 29,9$ & $\mathrm{p}=0,077$ & $125,7 \pm 27,5$ & $126,5 \pm 26,5$ & $\mathrm{p}=0,561$ & $\mathrm{p}=0,051$ \\
\hline $\begin{array}{l}\text { Apolipoproteína B } \\
(\mathrm{mg} / \mathrm{dl})\end{array}$ & $97,6 \pm 20,5$ & $99,2 \pm 11,5$ & $\mathrm{p}=0,855$ & $99,5 \pm 21,4$ & $106,1 \pm 15,3$ & $* p=0,046$ & $\mathrm{p}=0,165$ \\
\hline
\end{tabular}

* Diferenças estatisticamente significativas.

\section{Discussão}

De forma similar aos estudos anteriores realizados com contraceptivos de baixa dose contendo gestodeno ou desogestre ${ }^{4,6}$, os presentes resultados confirmaram a sua eficácia. Embora o Índice de Pearl não tenha sido calculado, uma vez que o tamanho da amostra foi dimensionado para avaliação de controle de ciclo, a única gravidez que ocorreu foi por falha de tomada de uma paciente no grupo desogestrel.

Considerando os dados relativos aos sintomas relatados pelas usuárias durante o tratamento, as duas preparações podem ser consideradas como bem toleradas, pois a porcentagem de mulheres que relataram um ou mais eventos adversos foi de aproximadamente $20 \%$. Os eventos mais freqüentes foram as queixas de desconfortos comumente associados aos tratamentos hormonais, tais como náusea, cefaléia e desconforto mamário. Além disso, apenas em 6 e 8 casos, respectivamente nos grupos gestodeno e desogestrel, houve abandono do tratamento motivado por aparecimento de efeito adverso.

Com base nos dados registrados nos diários de estudo, observou-se no grupo gestodeno melhora de sintomas pré-existentes tais como náusea, cefaléia, nervosismo, desconforto mamário, dor abdominal, secreção vaginal anormal e dismenorréia. No grupo desogestrel houve melhora do nervosismo, secreção vaginal anormal e dismenorréia. Considerando-se que os dados dos diários tinham por objetivo o seu registro segundo a percepção de sintomas pelas próprias pacientes ao longo do tratamento, o grupo gestodeno mostrou tendência mais favorável por ter sido associado à melhora de maior número de sintomas. Com relação à ocorrência de náusea, a tendência favorável que se verificou no grupo gestodeno foi mais marcante, uma vez que com desogestrel houve aumento significativo da incidência de náusea em relação ao pré-tratamento. A diminuição de apetite observada no grupo desogestrel poderia estar relacionada à presença de náusea.

O controle de ciclo foi adequado com as ambas preparações, não se observando casos de amenorréia e os episódios de sangramentos irregulares diminuíram ao longo do tratamento. Embora sem haver significância estatística, a diminuição da ocorrência de sangramento irregular foi mais precoce no grupo gestodeno. Nos dois grupos os sangramentos foram do tipo spotting na maioria dos casos. Considerando-se o total de ciclos, a incidência de sangramento de escape foi significativamente menor no grupo gestodeno $(4,6 \%$ dos ciclos) do que no grupo desogestrel $(8,1 \%)$. Estes resultados foram similares aos observados previamente em estudo ${ }^{3}$ que avaliou os efeitos do gestodeno e desogestrel associados a $30 \mathrm{mg}$ de etinilestradiol, onde a freqüência de ciclos sem sangramento de escape foi de $96,3 \%$ com gestodeno 
e 93,3\% com desogestrel.

Com relação aos resultados laboratoriais que avaliaram os parâmetros de coagulação e de fibrinólise, não se comprovou uma alteração que pudesse ser interpretada como tendo significado clínico. Nos dois grupos, os fatores que apresentaram alguma alteração estatisticamente significativa com relação aos valores no prétratamento permaneceram dentro dos limites da faixa de normalidade. Embora neste estudo os exames tenham sido realizados em um número limitado de mulheres e a padronização tenha também limitações de ordem prática, os resultados confirmam aqueles obtidos em estudos específicos anteriores $^{3,6}$, que não observaram modificações clinicamente significativas sobre o sistema hemostático com gestodeno ou desogestrel.

Com relação ao perfil lipídico, observou-se aumento dos níveis de HDL com o uso de ambas as preparações e aumento também da apolipoproteína B apenas no grupo desogestrel. Verificaram-se discretas alterações nos triglicérides (diminuição no grupo gestodeno e aumento no com desogestrel). A interpretação clínica desses resultados deve ser cuidadosa, pois o perfil lipídico foi avaliado em um número limitado de mulheres, durante apenas seis ciclos de tratamento e em uma faixa etária onde o perfil lipídico não é considerado um fator crítico de risco cardiovascular.

Com relação ao peso, não houve variação no grupo gestodeno, mas registrou-se aumento médio, estatisticamente significativo, de $1 \mathrm{~kg}$ no grupo com desogestrel, ao final de seis ciclos de tratamento. Em relação às mulheres que apresentaram aumento de peso, em cerca de $11 \%$ delas, nos dois grupos, este foi superior a $2 \mathrm{~kg}$. No grupo que empregou gestodeno, 26,6\% tiveram ganho de menos de $2 \mathrm{~kg}$ contra 51,4\% no grupo que usou desogestrel. Analisando-se esses resultados a respeito do ganho de peso conjuntamente com o que mostrou maior freqüência na perda de apetite no grupo com desogestrel, conclui-se que a manutenção de peso observada com o gestodeno poderia, de alguma forma, ser relacionada à ausência de retenção hídrica. Embora com intepretação clínica não bem definida, o gestodeno possui efeito antimineralocorticóide ${ }^{6}$, o que poderia, em alguns casos, estar associado à manutenção do peso, pois não ocorreria retenção hídrica. Estudo realizado com uma preparação contraceptiva contendo $75 \mathrm{mg}$ de gestodeno e $30 \mathrm{mg}$ de etinilestradiol ${ }^{6}$, que avaliou a composição corpórea de água e gordura ${ }^{5}$ durante o uso do contraceptivo, observou que, os casos de aumento de peso, seriam devidos ao aumento de gordura. No entanto, outros estudos são necessários para que se possa melhor interpretar a variação do peso durante o uso de contraceptivos orais.

\section{SUMMARY}

An open-label comparative study was conducted in nine centers in Brazil to evaluate the tolerability and cycle control of two low-dose oral contraceptives containing $20 \mathrm{mg}$ ethynylestradiol/75 mg gestodene and $20 \mathrm{mg}$ ethynylestradiol/ $150 \mathrm{mg}$ desogestrel, during six treatment cycles. A total of 167 healthy sexually active women were enrolled (77 in the gestodene group and 90 in the desogestrel group) and 138 completed the six-cycle treatment period. A lipid and hemostatic profile was performed for a subgroup of first users. A total of 867 cycles were evaluated. Irregular bleeding did not occur in $95.4 \%$ of the cycles evaluated with gestodene and in $91.9 \%$ with desogestrel. Tolerability was good with both preparations but there was significantly more nausea in the desogestrel group. Cycle control was good with both preparations with a significantly lower incidence of irregular bleeding with gestodene when all cycles were considered. There were no clinically significant changes in the hemostatic profile. Lipid profile showed a trend to be more favorable after six cycles of treatment with both preparations. Women in the gestodene group did not present changes in the mean weight; in the desogestrel group there was a significant mean weight increase of $1 \mathrm{~kg}$ after six cycles of treatment. Compliance with treatment was good with both preparations. Results of this study demonstrated that low-dose preparations containing gestodene or desogestrel combined with $20 \mathrm{mg}$ of ethynylestradiol are well-tolerated oral contraceptives that provide good cycle control.

KEY WORDS: Contraception. Low-dose contraceptive. Gestodene. Desogestrel. Cycle control. Estrogen. Progesterone. Coagulation. Lipid profile.

\section{Agradecimentos}

Agradecemos a: Maristela Seoanes Precivale pela análise estatística, Iolanda Fernandes de Oliveira pela monitorização clínica do estudo e Solange Cristina Davino pelo suporte bibliográfico. 


\section{Referências}

1.Endrikat J, Jaques MA, Mayerhofer M, Pelissier C, Müller U, Düsterberg B. A twelve-month comparative clinical investigation ot two low-dose oral contraceptives containing $20 \mathrm{mg}$ ethynylestradiol/75 mg gestodene and $20 \mathrm{mg}$ ethynylestradiol/150 mg desogestrel, with respect to efficacy, cycle control and tolerance. Contraception 1995; 52:229-35.

2.Latin American Oral Contraceptive Study Group. Clinical comparison of monophasic oral contraceptive preparations of gestodene/ethinyl estradiol and desogestrel/ethynyl estradiol. Contraception 1994; 50:201-14.
3.Melis GB, Fruzzetti F, Ricci C, Carmassi F, Fioretti P. Oral contraceptives and venous thromboembolic disease: the effect of the oestrogen dose. Maturitas 1988; Suppl 1:131-9.

4.Nevinny-Stickel J. German trial of an oral contraceptive containing $0.150 \mathrm{mg}$ desogestrel plus 0.020 mg ethynylestradiol. Acta Obstet Gynecol Scand 1987; 144 Suppl:19-21.

5.Reubinoff BE, Grubstein A, Meirow D, Berry E, Schenker JG, Brzezinski A. Effects of low-dose estrogen oral contraceptives on weight, body composition, and fat distribution in young women. Fertil Steril 1995; 63:516-21.

6.Wilde MI, Balfour JA. Gestodene - A review of its pharmacology, efficacy and tolerability in combined contraceptive preparations. Drugs 1995; 50:364-95. 